\title{
CPU card for Remote Terminal Unit of SCADA System for Traction Power Network of Railways
}

\author{
Shrilakshmi N.S. ${ }^{1}$ and Leelavathi G. ${ }^{2}$ \\ ${ }^{1}$ Research and Development, ITI Limited. Bangalore and ${ }^{2}$ Assistant professor, Govt.S.K.S.J.Technological \\ Institute,Karnataka,Bangalore,india.
}

\begin{abstract}
SCADA stands for Supervisory Control And Data Acquisition. SCADA system is employed all over the world for effectively monitoring and controlling different utility networks.

Indian Railways employs SCADA systems for monitoring and controlling the distribution of Traction Power. The systems employed were of proprietary in nature and did not have any standard specifications both with respect to protocol and hardware. Moreover, the SCADA systems from different vendors could not coexist in a given network. In view of this, Railways formulated the specifications for the SCADA systems wherein the systems should conform to SPORT protocol for data transaction and corrective action of tripping the circuit breakers in case of over load etc. In current scenario, lots of intelligence needs to be built into the Remote Terminal Unit, which requires a powerful Central Processor Unit (CPU). The objective of this paper is to present the design and development of the CPU Card for Remote Terminal Unit of SCADA system for Indian Railways. The traction power distribution network requires continuous real time monitoring and controlling of the system failing which, leads to catastrophic disasters. SCADA system helps in achieving real time monitoring. This new CPU card design meets all the high demands of the Indian Railways.
\end{abstract}

Keywords: SCADA, SPORT, CPU, RCC, RTU \& TPN

\section{INTRODUCTION}

SCADA system is generally used to automatically monitor and control the various operations in the utility networks ${ }^{[4][7]}$, where manually doing is difficult and error prone.

The Traction power network of Railways supplies power $(25 \mathrm{kV}, 1 \Phi, 50 \mathrm{~Hz})$ for electric trains to operate. This is a very huge network spread across the country. As the electric trains traffic has increased enormously these years, this power network requires continuous real time monitoring of the traction power, failure of which may lead to untoward incidents. Real time monitoring ${ }^{[5][6]}$ of the Traction power network consisting of transformers, circuit breakers etc., can be efficiently done using the SCADA system. With the advancement in computer technology and latest microprocessors /microcontrollers, present SCADA system provides an entire view of the network with each device details and the controlling of any part of the network can be done with the click of a mouse on the GUI of the control centre.

The SCADA system for Railways traction power network ${ }^{[1]}$ consists of two major entities Remote Control Centre (RCC) and Remote Terminal Units (RTUs). The RCC does the main supervision and controlling activity of the network through RTUs. As the power network is very huge, several RTUs are required for interfacing with the network elements. These RTUs are controlled by RCC.

End devices to be monitored are interfaced to RCC through the RTU. The RTU polls the end devices \& collects the status/alarm information of the end devices, stores it, and uploads to RCC when queried. The information collected from RTUs is maintained in the database and is displayed through GUI at RCC. The collected data is analyzed and used for network controlling activity. The RCC configures and controls the RTUs according to the network requirement which can be dynamically viewed on GUI.

This paper presents the design and development of CPU card for the RTU which is the brain behind all the activities of RTU. The CPU card which is based on the ARM microcontroller interfaces with peripheral cards which communicate with end devices.

\footnotetext{
II. LITERATURE SURVEY

Traction power distribution of railways ${ }^{[1]}$

To understand the railway Traction Power Network the GR issued by Railways is used. This document clearly explains the network, requirements, configuration, control, testing and monitoring. On the basis of this GR the SCADA system and the CPU card are designed.
} 


\section{Study of general SCADA system}

SCADA system has been widely used all over the world for various distribution networks. Various SCADA systems $\mathrm{s}^{[3][4]}$ were studied to know the their functionality and requirement ${ }^{[2]}$. By this study, it was understood that all the SCADA systems in general have the common requirement of monitoring and controlling of the end devices and logging of the reports of the status and system activities. The major difference between various SCADA systems ${ }^{[5]}$ was the transducers used for interfacing of the end equipments. The scheme of the SCADA system was studied and it was planned to develop SCADA system to suit the traction power distribution network of railways.

\section{IEC 60870-5 protocol standard ${ }^{[9]}$ :}

IEC 60870 part 5 is one of the IEC 60870 set of standards which define systems used for telecontrol (supervisory control and data acquisition) in electrical engineering and power system automation applications. Part 5 provides a communication profile for sending basic telecontrol messages between two systems, which uses permanent directly connected data circuits between the systems. The IEC Technical Committee have developed a protocol standard for telecontrol, teleprotection, and associated telecommunications for electric power systems, which is IEC 60870-5.

\section{OVER VIEW OF THE RAILWAYS SCADA SYSTEM.}

Traction power network of Railways ${ }^{[1]}$ consists of elements like circuit breakers, transformers etc., whose status/alarms needs to be monitored in real time. All the points of the end devices to be controlled in a traction power network are brought to a control panel in each railway substation. Each RTU is connected to the railways control panel of that sub-station.

\section{Remote Control center (RCC)}

The RCC does the controlling and monitoring of the network through RTUs. RCC consists of a network of computers i.e., Servers and Workstations each doing a dedicated job. Separate computers are used as Front End Processors (FEP) to collect data from Remote Terminal Units (RTU).

There is continuous exchange of messages between the FEP and the RTUs and the FEP initiates this communication activity. The FEP after acquiring the data from various RTUs passes the same to server. The server processes the received raw data and stores it in the data base. The MMI application uses the data from the database for displaying the status of the network.

MMI is the user friendly interface and it displays each and every physical description of the device graphically on the screen. Authorized user on the MMI can check and control the working of each and every device and hence the system is secured ${ }^{[7]}$. MMI is a set of applications that displays the status of traction power distribution network to operators with all the details of the network devices. Through the user friendly GUI, operators can monitor, analyze and control the substation equipment using MMI.

\section{Remote Terminal Unit (RTU)}

Remote Terminal Unit (RTU) allows the RCC to control and monitor the sub-stations of traction power network. RTU is deployed in each sub-station and interconnected to the sub-station end equipments to facilitate the Telecontrol, Telesignal and Measurand features of SCADA system.

All the modules of RTU are physically equipped inside a cabinet including the relays for telecontrol / telesignal interface and transducers for PT/CT/PF interfaces. The RTU system design employs standard plug in type printed card modules. The number of modules sitting on the common backplane depends on the number of parameters to be controlled and monitored in the substation. The modules sitting on the backplane (Mother board) will be CPU, Digital output, Digital Input and Analog Input modules. The CPU card communicates to peripheral cards on RS 485 bus and on RS232 bus with modem. The functionality of the various components of the RTU is as described below.

\section{CPU Card}

CPU card is responsible for the major activities of the RTU. It communicates to RCC through modem on one end and talks to peripheral cards on the other end to achieve SCADA functionalities of RTU.

\section{Digital Output Card (DO Card)}

DO card interacts with CPU card for control selection and execution. DO card supports 16 controls for the $\mathrm{ON}$ and OFF operation of end-devices. DO card receives control signals from the CPU card and extend the control signals to series of relays which in turn control the end devices. It facilitates select-check-execute feature of control function to avoid any wrong command being executed. Plug-in relays are used as interface for ON / OFF operation by extending +110VDC to close/trip coil of end-devices. 
Digital Input Card (DI Card)

CPU card interacts with DI card for tele-signal monitoring. DI card supports 32 tele-signals with relays operating at 24VDC. All the telesignals are provided at 110VDC on the control panel. Interface cards are used to translate $+110 \mathrm{~V}$ received from railway control panel to $+24 \mathrm{~V}$ signals which are then fed to DI cards. This information is stored and sent to the CPU card during polling.

\section{Analog Input Card (AI Card)}

CPU card interacts with AI card for measurand monitoring i.e., analog data like traction voltage, current and power factor. AI card supports 8 A/D channels. The ADC facility in the ARM controller facilitates the conversion of analog to digital signals. This digital information is sent to the CPU card when polled. Transducers are used to convert the analog inputs of end-devices to an equivalent output of 0-10VDC to AI card.

\section{Modem}

FSK Modem at 600/1200 Baud is used for the communication between RTU \& FEP.

\section{Power Supply Unit (PSU)}

Power supply unit works on $230 \mathrm{VAC}$ and generates the required DC voltages $3.3 \mathrm{~V}, 12 \mathrm{~V} \& 24 \mathrm{~V}$ required for the system.

\section{CPU CARD INTERNAL ARCHITECTURE AND SPECIFICATIONS}

Aim of this paper is to present the design of the CPU card for the RTU. The CPU card is the heart of the Remote Terminal Unit. It is responsible for the collection of the telesignals, measurands from the end devices and to control the end devices as per the commands issued by RCC.

The CPU card in RTU does the following activities.

- Communicates to RCC through modem on one end and talks to peripheral cards on the other end.

- Collects alarms \& device status through Digital Input Card.

- Collects Analog measurand data of the network through Analog Input Card.

- Issues Control commands to end devices through Digital Output Card.

All the cards are connected through motherboard on RS485 bus at $19.2 \mathrm{kbps}$. The CPU in the RTU scans Digital Input cards and the Analog Input cards at a rate of $200 \mathrm{~ms}$ and stores the change of status as well as the analog data in the memory. Whenever RTU receives a query from the master station, it transfers the stored data in the CPU to the master station. CPU card communicates with FEP at RCC through a modem \& responds only if the station address is matching.

LED indications are provided on CPU \& peripheral cards for easy diagnostics of RTU. Also one RS232 port is provided on the front panel of the CPU card for the diagnostic test of CPU as well as all the peripheral cards.

\section{DESIGN PRESENTATION}

The storage and interfacing requirements of the RTU are provided by CPU card. The RTU requires four UART interfaces and one Ethernet interface for future use. UART interfaces are used for debugging, communication with modem \& peripheral cards and a spare port for future use. One JTAG interface is provided for down loading software.

32 bit ARM7 Microcontroller (LPC 2378, Manufactured by NXP) is used which provides 512kB Flash \& $32 \mathrm{kB}$ SRAM for software and data storage. External Reset circuit is provided in the CPU card for extending self and peripheral cards reset.

GPIOs of ARM controller are used for:

1. Visual indication of communication with modem and peripheral cards.

2. Self address identification of RTU.

3. Setting the baud rate for communication with modem.

Buffers are provided for isolation and drive the LED and other circuitry. RS232 level converters are provided for communicating with modem and display terminal. RS485 interface is provided for multi-drop connectivity through the mother board for communication with peripheral cards. Half Euro (32 pin) and Full Euro (64 pin) connectors are used for connectivity between cards and Mother Board, for sturdy connection to with stand heavy vibration due to train movements near the cabins where RTUs are installed. $12 \mathrm{MHz}$ crystal provides the necessary clock for the function of the in-built oscillator of the ARM controller. Once in $200 \mathrm{~ms}$ the CPU card polls the peripheral cards to get the status information. 
CPU card uploads the stored information to RCC when polled by it. Watch dog timer prevents the software from hanging. RTC is used for real time display and synchronization with RCC. Other timers are used for providing the required delays for proper working of software. Station address setting facility is provided in CPU to address 255 stations by FEP. CPU accesses the peripheral cards through RS485 bus on the motherboard and facilitates the control and monitoring functions of SCADA systems.

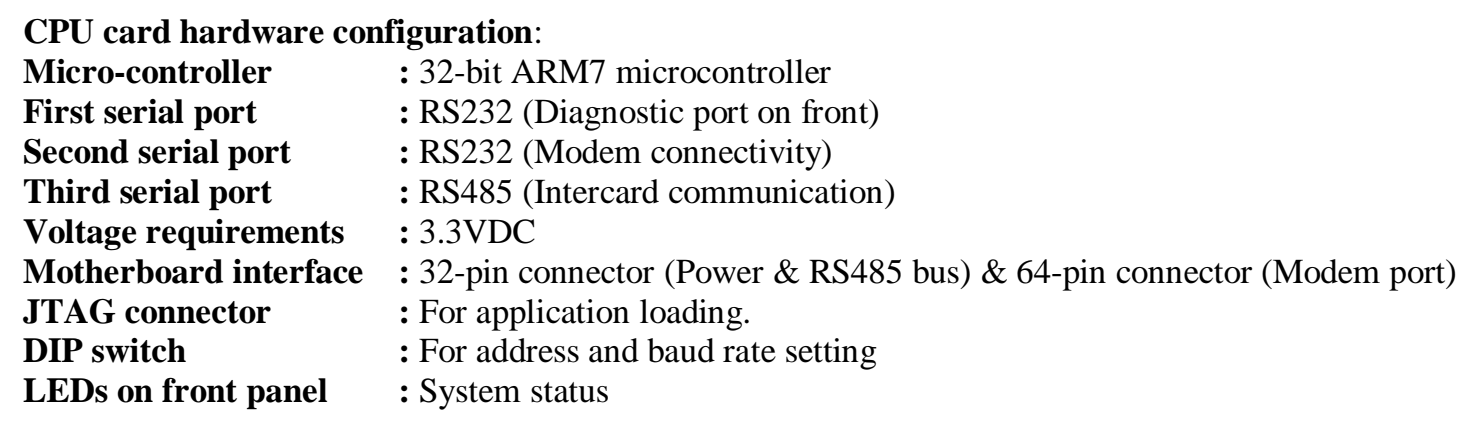

\section{Software implementation}

The communication between FEP of RCC and CPU card of RTU is through the SPORT protocol. The frame format of the SPORT protocol consists of Block 0, Block1, .... Block n depending on the size of the message and block 0 is mandatory in all the messages. The frame format chosen is FT2 type of IEC standard IEC-870-5.

The software for the CPU card activity is depicted in the flow chart as shown below in Fig. 1. Any command from RCC will be given top priority and will be executed immediately halting the normal polling activity. Also request for debugging will be continuously monitored and will be serviced immediately as shown in the flow chart.

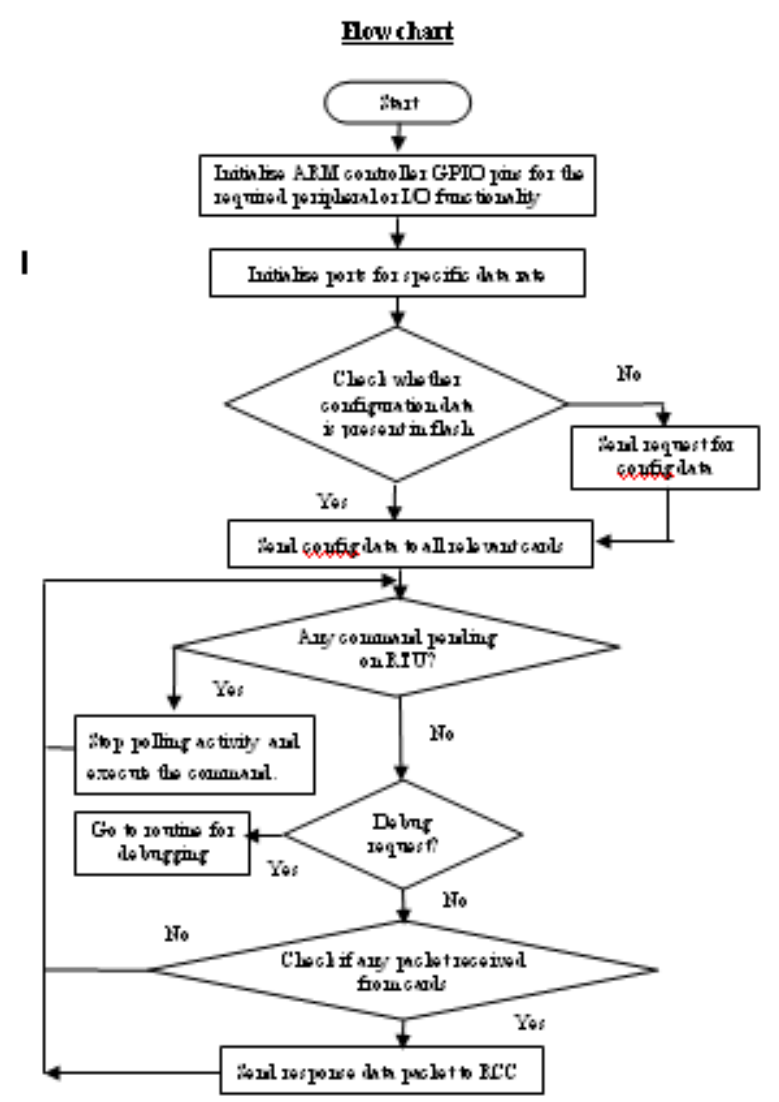

Figure 1: CPU functionality flow chart

VI. CONCLUSION \& FUTURE WORK

The CPU card is designed for the specifications of railways SCADA system. Hardware specifications meet the requirement of railways with state of the art technology, required capacity, and connectivity with various types of peripherals, real time operation and diagnostic capabilities. 
Communication between RCC \& RTU is as per SPORT protocol as specified by Railways which aids in error free communication.

The CPU card is tested in both standalone mode and in integration mode with peripheral cards of the RTU. The communication with RCC is tested as per SPORT protocol. It is confirmed that the results are in conformance with the specifications of the railways.

The present CPU card designed is for the railways and meets exact requirements of RTU of power traction network for both Hardware and software specifications and also network conditions to withstand the vibrations produced by the movement of trains and also the two wire communication network existing at present. The designed card uses voice band modem for communication between RCC \& RTUs. The data transfer rate can go up to 9600 baud. The design concept presented in this paper can be used with minimum changes, in various other SCADA systems like water distribution network, electricity distribution network, gas distribution network etc., by changing the transducers according to the requirement.

Process control or SCADA system can be done with IP networks ${ }^{[8]}$ where the speed is limited to the capacity of the IP connection.

In an IP network, communication between RCC \& various RTUs can be done simultaneously to reduce the response time of overall system and enhance the performance of the system as IP networks can provide enhanced reliability, while ensuring data protection, back up and recovery. The Ethernet connectivity provided in the CPU extends the use of SCADA system to huge networks and also increase the efficiency of the system.

\section{REFERENCES}

[1] Generic requirement (GR) specification issued by RDSO (Research and Development Standards Organization) of Indian railways.(Specification No: TI/SPC/RCC/SCADA/0990

[2] Tender document of Guntur Municipal Corporation for SCADA system for water distribution network.

[3] I. F. Akyildiz, W. Su, Y. Sankarasubramaniam and E. Cayirci, "A Survey on Sensor Networks" IEEE Dr. Aditya Goel \& Ravi Shankar Mishra, International Journal of Engineering (IJE), Volume (3): Issue (1) 65 Communications Magazine, Vol. 40, No. 8, pp. 102-114, August 2002.

[4] Hosny A. Abbas \& Ahmed M. Mohamed "Review on the Design of Web Based SCADA Systems Based on OPC DA Protocol" International Journal Of Computer Networks (IJCN), Volume (2) : Issue (6) page No.266

[5] Son,S.H.,Beckinger, R.C.,Baker,D.A. "Overview of Real Time Database Management System Design for Power System SCADA System", Proceedings of the IEEE, 2005.52:62-66

[6] L. Wiliem a, *, D.Hargreaves a, R.F. Stapelberg b, P.K.D.V. Yarlagadda "Development of real-time data filtering for SCADA system" Journal of achievements in materials and manufacturing engineering, volume21, issue2, April 2007, Pages 89-93.

[7] E. Dagle (SM), S.E. Widergren (SM) and J.M. Johnson (M) "Enhancing the Security of Supervisory Control and Data Acquisition (SCADA) Systems: The Lifeblood of Modern Energy Infrastructures" Power Engineering Society Winter Meeting, 2002. IEEE Volume 1, Issue, 2002 Page(s): 635 vol.1

[8] McClanahan, R.H., "The Benefits of Networked SCADA Systems Utilizing IP Enabled Networks", Proc. Of IEEE Rural Electric Power Conference 5-7 May 2002 Pages: C5 -C5_7Technical Information Bulletin 04-1, Supervisory Control and Data Acquisition (SCADA) Systems, NCS TIB 04-1, Oct. 2004

[9] IEC 60870-5 Protocol Standards

[10] KEIL $-\mathrm{uVision} 3.0$ developers guide. 